\title{
Improvements of Cloud Particle Sizing with a 2D-Grey Probe
}

\author{
ANDREAS REUTER \\ GKSS Forschungszentrum Geesthacht GmbH, Institut für Atmosphärenphysik, Geesthacht, Germany \\ STEPHAN BAKAN \\ Max-Planck-Institut für Meteorologie, Hamburg, Germany \\ 31 January 1996 and 17 October 1997
}

\begin{abstract}
The potential of the 2D-Grey optical array probe (OAP) (with $10-\mu \mathrm{m}$ resolution) to determine cloud microphysical properties is studied. Systematic test measurements with a spinning glass disk with sample spots of various sizes between 50 and $500 \mu \mathrm{m}$ in diameter were conducted. These measurements show that the particle image diameter increases considerably if the particle crosses the illuminating laser beam at increasing distance from the object plane. Eventually, shadow images of the smaller spots lose even their circular image shape and appear fragmented. A method is proposed to improve the estimation of the nominal particle size of droplets from the recorded image by exploiting the four available shadow (grey) levels. Laboratory tests show that spherical particles from 50 to $500 \mu \mathrm{m}$ in diameter can be properly sized with an rms uncertainty of less than $6 \%$. After discussion of the concept of depth of field in OAPs, a definition for the 2D-Grey probe is presented that is consistent with the standard definition for the 2D-C probe. The authors' measurements show the depth of field of the 2D-Grey probe to be three times larger than the value conventionally assumed for the $2 \mathrm{D}-\mathrm{C}$ probe for which similar corrections have been recently discussed in the literature. Finally, the impact of these findings on particle size distribution for in situ measurements is discussed.
\end{abstract}

\section{Introduction}

The global climate system depends crucially on the properties of clouds and cloud systems, which have a major impact on chemical cycles and on the atmospheric radiation balance (Stephens et al. 1990; Slingo 1990). The ability to understand this impact depends on information about 3D structure and temporal variability, together with knowledge of the microphysical properties (Albrecht 1989). While aspects of cloud structure and distribution have been extensively studied by surface observers and satellite image analysis techniques, the remote determination of cloud microphysical properties is still under development and requires extensive validation information from direct measurements within the clouds. In addition, the vertical and horizontal variability of cloud microphysical properties, especially on a smaller scale, cannot yet be derived satisfactorily from remote measurements. Cloud microphysical properties therefore have to be measured in situ with appropriate airborne instrumentation.

For this purpose, the PMS (Particle Measuring Systems, Inc., Boulder, Colorado) series of optical probes

Corresponding author address: Dr. S. Bakan, Max-Planck-Institut für Meteorologie, Bundesstrasse 55, D-20146 Hamburg, Germany. E-mail: bakan@dkrz.de for the derivation of particle size distributions have been widely used. For particles larger than about $50 \mu \mathrm{m}$, the optical array probes (OAPs) provide shadow images for the estimation of size and shape. The standard 2D-C probe usually records binary images of cloud particles at a resolution of $25 \mu \mathrm{m}$. The latest model is the socalled Grey probe, a version of the imaging probes with a 2-bit (= four grey levels) resolution of the array illumination intensity, which is also available with 10$\mu \mathrm{m}$ size resolution. The present paper refers to this probe type, which we employ for studies of the onset of drizzle (similar to Martin et al. 1994) and of microphysical properties of cirrus.

Unfortunately, problems arise with the blurred images of smaller particles that pass the OAP system out of the object plane, leading to erroneous particle sizing. This problem, plus that of incomplete knowledge of the correct depth of field for the OAP 2D probes, has been mentioned in the literature (Heymsfield and Baumgardner 1985; Joe and List 1987). Korolev et al. (1991) studied the imaging behavior of OAP probes with the help of Fresnel diffraction calculations of opaque disks and with detailed measurements. They concluded that the particle diameter may be overestimated by up to $85 \%$ with the $2 \mathrm{D}-\mathrm{C}$ probe due to erroneous interpretation of out of focus particle images. The present paper documents the imaging behavior of the Grey probe from 
TABLE 1. Grey-level denomination.

\begin{tabular}{cc}
\hline \hline $\begin{array}{c}\text { Grey- (shadow) level } \\
\text { denomination }\end{array}$ & $\begin{array}{c}\text { Reduction of array illumination } \\
\text { intensity of unshadowed }(\%)\end{array}$ \\
\hline NONE & $0-25$ \\
MIN & $25-50$ \\
MID & $50-75$ \\
MAX & $75-100$ \\
\hline
\end{tabular}

measurements with opaque disks (section 2). A procedure is proposed to recover real cloud droplet diameter by exploiting the available gray-level information (section 3), and detailed measurements of the depth of field are provided (section 4 ).

\section{The 2D-Grey cloud probe}

\section{a. General description}

In the PMS optical array probes, an appropriately widened laser beam illuminates a linear detector array. Particles crossing the part of the beam that is exposed to the airflow cast a shadow onto the detector array, which is recorded if the signal intensity at the array element is reduced below some threshold value. Consecutive shadow slices are then combined in the OAP2D(2)-C to a two-dimensional binary particle image (Knollenberg 1970).

The most advanced version, the 2D-Grey cloud probe (OAP-2D-GA2), contains a photo diode array of 64 elements and zoom optics that enable resolutions down to $10 \mu \mathrm{m}$ along the array. Due to the maximum slice rate of $5 \mathrm{MHz}$, a $10-\mu \mathrm{m}$ resolution in-flight direction is achieved at true air speeds of $50 \mathrm{~m} \mathrm{~s}^{-1}$ or less. Also, because $5 \mathrm{MHz}$ is the maximum slice rate for the 2D$\mathrm{C}$, and the 2D-Grey probe has to transfer additional grey-level information to the data acquisition system, the 2D-Grey has a lower sample rate than the 2D-C when in high concentrations of smaller particles. This limitation can affect sample volume and particle sampling statistics of the 2D-Grey probe compared to the 2D-C.

The 2D-Grey probe enables the distinction between four shadow (grey) levels per image element, corresponding to thresholds at $75 \%, 50 \%$, and $25 \%$ of the laser illumination intensity. The distinction is accomplished by three comparator steps that compare the actual array element illumination with the unshadowed value. The naming of these grey levels is specified in Table 1. By this nomenclature, MIN is the lightest grey level with the smallest decrease of the laser intensity, whereas MAX is the darkest level.

During the process of adopting the analysis technique for $2 \mathrm{D}-\mathrm{C}$ probe data to the 2D-Grey probe, some general problems with OAPs became obvious. Ideally, each particle crossing the laser beam causes an image that is recorded by the data acquisition system. It is not known a priori how the shape and size of the image are related

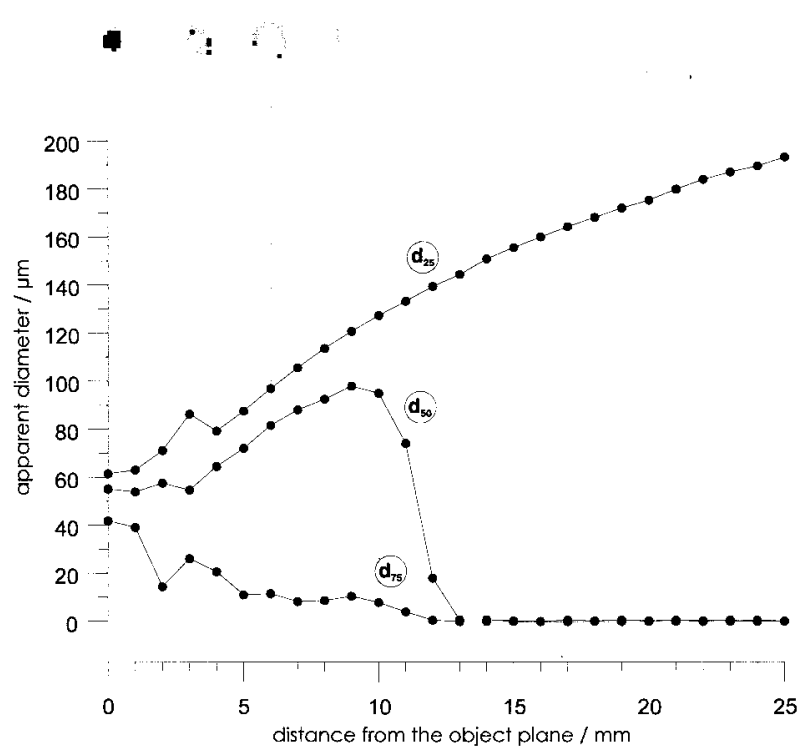

FIG. 1. Image width for the $25 \%, 50 \%$, and $75 \%$ shadow-level threshold vs offset from the object plane for circular spots of $50-\mu \mathrm{m}$ diameter. In addition, examples of particle images are shown at six distances from the object plane.

to the particle itself. This problem has to be solved to determine the particle size on the basis of the particle image.

\section{b. Depth of field in OAPs}

The calculation of meteorological parameters, such as liquid water content and number density, from OAP images is based on the sampling volume of the probe. Besides the true airspeed of the aircraft and the array width projected into the sample volume, this requires the knowledge of the effective sampling interval along the laser beam between the probe tips.

In OAPs the object plane is usually located in the middle between the probe tips, which are $61 \mathrm{~mm}$ apart. Particles passing the laser beam through the object plane cast a sharp, in-focus shadow image on the detector array. However, particles passing to either side of the object plane appear blurred in the image (array) plane. The variation of the apparent image diameter with distance from the object plane is demonstrated in Fig. 1. This figure shows several images of an opaque spot of $50-\mu \mathrm{m}$ diameter at different distances from the object plane recorded with the $2 \mathrm{D}-\mathrm{Grey}$ probe (for details see section 3). The most striking feature is the dramatic increase of the image diameter. At large distances from the object plane the image shape no longer resembles the circular shape of the particle and eventually, only fragments of the image remain. While the eye is mostly guided by the outline of the barely shadowed (MIN) image elements, the medium shadowed (MID) image elements exhibit a similar change with distance from the object plane, but the distance of maximum image 
size is nearer to the object plane. The number of completely shadowed (MAX) pixels, however, decreases with distance from the object plane. The small difference of the apparent diameter for the three shadow levels in the object plane results from the digitization by the finite size image array elements. Nevertheless, the diameter of the in-focus shadow image represents a fairly accurate measure of the size of the opaque spot.

The definition of an appropriate sampling interval along the laser beam of a 2D-C probe has to rely on only one intensity level. Knollenberg (1970) found experimentally that a threshold of $40 \%$ of the laser intensity in OAPs defines the distance along the laser beam within which all recognizable images originate and the size of all particle images differ from the true particles size by less than about $10 \%$. This result seems also plausible from Fig. 1 if it is kept in mind that the 40\% threshold corresponds to $60 \%$ reduction in array illumination. He called this distance "depth of field" and found a quadratic dependence on particle size. If $l_{\mathrm{DOF}}$ refers to the total sampling interval along the laser beam, not just to the distance from the object plane, the corresponding relation with particle diameter $d$ may be written as

$$
l_{\mathrm{DOF}}=\frac{3 d^{2}}{2 \lambda},
$$

where $\lambda$ denotes the laser wavelength of $632.8 \mathrm{~nm}$.

The term depth of field originates from photography, where it describes the distance in object space over which the optical system delivers an acceptably sharp image. How much indistinctness is tolerable is related to the viewing conditions as well as the ability of the observer's eyes to differentiate the fine details in an image. In particle probes with a fixed threshold level the indistinctness leads to the vanishing of all image pixels at some distance from the object plane, which is, consequently, called depth of field. This definition seemed a natural extension of the classic photographic concept for Knollenberg (1970), who analyzed photographic prints of the diffraction images with spatial resolution limited only by the grain size of the photographic print. The concept was eventually applied to real particle probes with finite array resolution. This resulted in the assumption that any visible image with at least one pixel triggered represents uniquely a real particle with a diameter near to the image diameter. While this would be true (within less than about $10 \%$ error in diameter) for the originally proposed $40 \%$ threshold level (i.e., $60 \%$ reduction of array illumination intensity), it is not true for most of the $2 \mathrm{D}-\mathrm{C}$ probes with the usual $50 \%$ threshold. As Fig. 1 indicates, at this level a certain image size is not uniquely related to a particle but can be caused by various sized particles at different distances from the object plane. Notwithstanding this difficulty, the findings from Knollenberg (1970) are often applied to the evaluation of OAP data. Heymsfield and
Baumgardner (1985) suggest evidence that the 2D-C probe responds to particles up to distances from the object plane that are two to three times $l_{\mathrm{DOF}}$ as defined by (1), although they do not doubt that the particles are accurately sized by their image. Joe and List (1987) also realized problems when using a Grey probe. They proposed to limit the distance from the object plane such that the image size again represents the real particle size within some narrow tolerance.

To avoid the problems with ambiguous relations between particle images and real particles in the 2D-C probe, Korolev et al. (1998) essentially propose to avoid the treatment of individual particle images but to calculate the probability for an image to represent a certain sized particle. They propose an inversion procedure to calculate the real size distribution from the size distribution of an ensemble of images. As this ensemble contains any recorded image, however small it is, this procedure is based on the definition of the depth of field for each particle size as that distance from the object plane, within which at least one pixel is shadowed.

Another approach is to use additional particle image features to improve the correct sizing of the individual particles. Such additional information is provided by the 2D-Grey probe through the availability of two additional shadow levels. In the present paper a technique is proposed to use the relative abundance of different grey levels in the image as a measure of the indistinctness of the image. This allows another unique definition of a depth of field as well as provides an almost unique way of inferring the real particle diameter from its image.

The objective definition of indistinctness in particle probes is, of course, somewhat different from its original more subjective meaning in photography. Nevertheless, the definitions are similar enough to warrant the use of the term depth of field also for the sampling distance along the optical axis of the OAP system. We suggest to combine the various depth of field definitions put forward by different authors into the following three requirements: 1) The extent of the depth of field must be known for all detectable particle sizes, that is, it must be well defined; 2) it must be possible to determine whether a particle passed the probe in- or outside the depth of field; and 3) there must be a procedure available, analytical or statistical, of calculating particle size on the basis of the related image information for all particles within the depth of field to an acceptable accuracy.

Note that results from the application of these criteria to OAP probe data may depend on the detailed definitions of particle image size (Korolev et al. 1998). Also the details of any resulting procedure may have to be changed between laboratory tests and real flight applications due to the finite response time of the probe electronics and in case of nonspherical particles. Work to clarify the problem of response time is under way. 


\section{Droplet image analysis for the 2D-Grey cloud probe}

An experimental approach was used to examine the imaging behavior of the 2D-Grey probe for spherical particles. Due to difficulties in generating water droplets in the size interval between 10 and $100 \mu \mathrm{m}$ with a wellcontrolled diameter, we decided to study the imaging behavior of the probe with the help of opaque spots on a spinning glass disk. This is justified since the diffraction pattern of a sphere and an opaque spot of equal cross section are equal for particles with large diameters compared to the laser wavelength (Korolev et al. 1991). However, this approach ignores the refracted component of light from a real droplet, which acts as a short-focus collecting lens. When the droplet is near the object plane, its focal point is imaged to the center of the particle image, where it adds to the Poisson spot of the diffraction pattern. Korolev et al. (1991) attribute part of the differences between the observed and calculated brightness pattern near the image center of glass beads to this effect. The additional intensity from this lensing effect increases with the square of the particle diameter, so that it makes a considerable contribution to the brightness of the central pixel for any droplet diameter larger than about $50 \mu \mathrm{m}$. Lawson (1996, personal communication) informed us about test measurements with $200-\mu \mathrm{m}$ polystyrene beads in which the brightness at the image center exceeds the ambient level of the background laser light by more than a factor of 2 . At a distance from the object plane, the droplet's focal point is no longer focused to the central pixel of the particle image. Redistribution of the additional intensity to an increasing number of pixels around the image center results in a decrease of the pixel intensity by the square of the distance from the object plane. Although we had no opportunity to study the implications of this lensing effect in detail, we conclude from all available evidence that it will only introduce minor errors and can therefore be ignored in our study of improved particle sizing for the following reasons. First, it is limited to the center of the droplet image with only slight change in the number of pixels at any of the shadow levels. Second, the additional intensity per pixel reduces rapidly with distance from the object plane, while there is no sizing problem near that plane. And third, the importance of this effect increases with particle size, while the sizing problem becomes smaller.

Therefore, measurements with a spinning glass disk with opaque spots of 50-, 60-, 70-, 80-, 100-, 120-, 150-, 200-, 300-, and 500- $\mu \mathrm{m}$ diameter have been performed for the present study. The spots were arranged on different concentric rings on the disk with 72 identical spots on each ring. Micropositioners were used to move the disk through the depth of field and between the different rings of spots (Fig. 2). This equipment was used to study the images of all available spot sizes at 10 positions between the probe tips. For mechanical

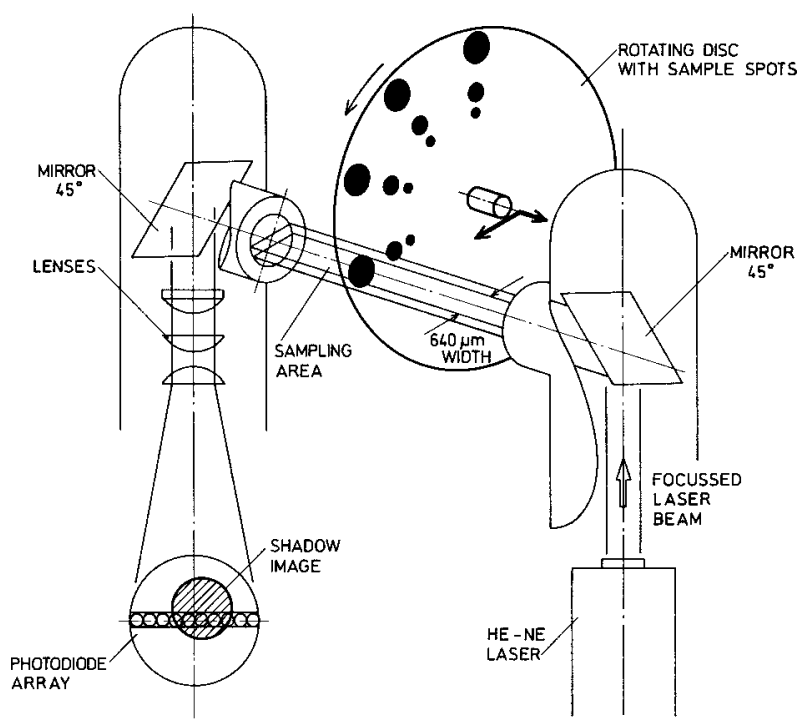

FIG. 2. Diagram of the test setup with a spinning glass disk cutting through the laser beam.

reasons, only $44 \mathrm{~mm}$ of the available $61-\mathrm{mm}$ distance between the probe tips could be spanned with the experimental setup. Special care was given to determining the exact position of the object plane, as this is the reference position for all measurements.

Before analyzing the measurements, invalid images caused by dirt on the disk or air bubbles in the glass layer had to be rejected. This was done with the help of a neural network classifier consisting of an ordinary three-layer back-propagation network. In the first step, occasional diffraction fringes were eliminated by using an image filter to remove pixels with very few neighbors (as in narrow lines). A projection of the resulting image onto the diode array was generated such that for each photo diode the maximum shadow level found was assigned to the diode element. In addition, a cut through the image along diode element 32 (center element) was extracted, utilizing the fact that all the valid images cast a shadow on this specific element due to the layout of the disk and the adjustment of the spots in the middle of the array. This combined information was used as the input for the network. The accuracy of the rejection process was determined by an independent sample of manually classified images and was found to be $93 \%$. A sample of 500 valid images per spot size and position between the probe tips has been used for the study of the imaging behavior of the 2D-Grey probe.

Figure 1 shows the apparent diameter of a $50-\mu \mathrm{m}$ spot at various distances from the object plane for the three threshold grey levels, $25 \%, 50 \%$, and $75 \%$, together with a few representative images. The diameter is derived as the maximum distance across the particle image in any of the recorded image slices for each grey level and is labeled $d_{25}, d_{50}$, and $d_{75}$, respectively. This definition of the image diameter $d_{50}$ is similar to the 


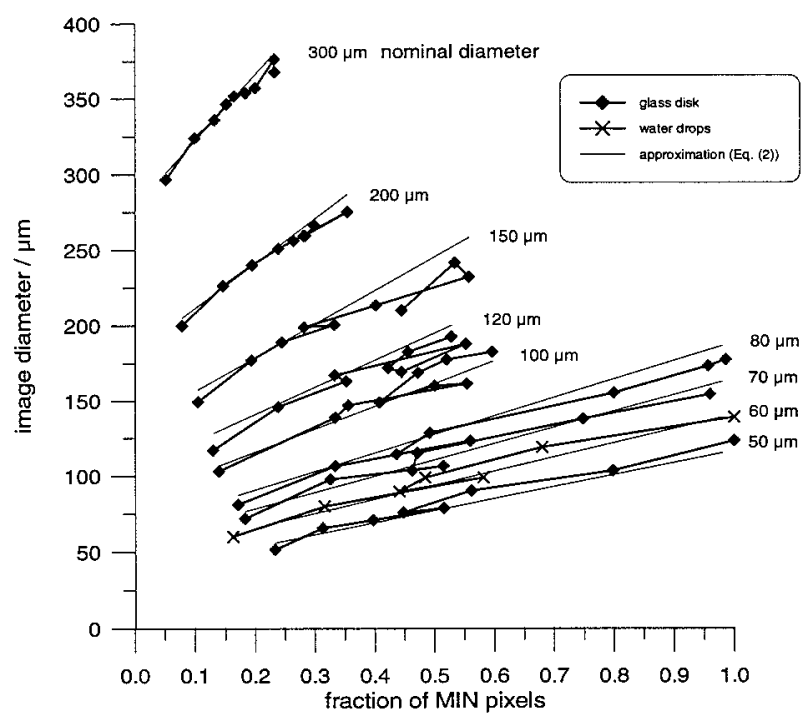

FIG. 3. Apparent particle image diameter $d_{25}$ vs fraction of pixels at the lowest gray level $q_{\mathrm{MIN}}$.

"width" of the shadow recorded by the 2D-C probe. The displayed variation of image size with distance from the object plane has been discussed in section 2 and is representative for all spot sizes. The slightly different diameter values in the vicinity of the object plane are due to the gray-level discretization.

This variability of the three shadow levels with distance from the object plane behaves in a systematic way for each test spot size. In particular, the ratio $q_{\mathrm{MIN}}$ of the number of MIN pixels to the total number of shadowed pixels (MAX + MID + MIN) increases systematically with distance from the object plane. The ratio $q_{\text {MIN }}$ essentially represents the degree of blurring of a particle image, which increases with increasing distance from the object plane. As this increase is different for different spot sizes, this quantity can be used to characterize the true particle size.

The variation of the apparent diameter $d_{25}$ with the fraction of pixels at the lowest grey level $q_{\text {MIN }}$ (taken from measurements at different distances from the object plane) is plotted for different particle sizes in Fig. 3 . The apparent image diameter generally increases with $q_{\text {MIN }}$ at a rate that is proportional to the nominal spot diameter. The smallest value of each of these curves represents the measurement in the object plane. Although this image contains mostly pixels at the MAX shadow level, there are still a few even at the MIN level along the image perimeter due to partial pixel illumination, causing $q_{\text {MIN }}>0$. As the relative importance of perimeter pixels increases with decreasing spot size, the smallest observed value of $q_{\text {MIN }}$ does so as well. For spot sizes smaller than about $90 \mu \mathrm{m}$, the largest value of $q_{\text {MIN }}$ is 1 , as the images of these particles do not contain any MAX or MID pixels beyond some distance from the object plane (as was shown in Fig. 1 for a 50$\mu \mathrm{m}$ particle). For larger spot sizes the number of MAX

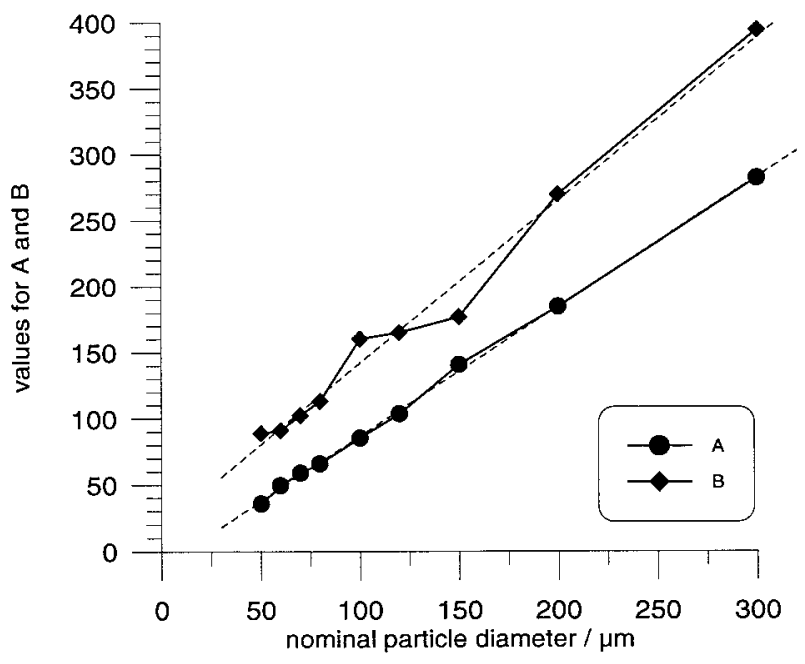

FIG. 4. Variation of slope $A$ and offset $B$ of the linear approximations of Fig. 3 vs nominal spot size and corresponding linear approximation of both coefficients.

and/or MID pixels no longer vanishes at any position between the probe tips and, therefore, the maximum value of $q_{\mathrm{MIN}}$ remains less than 1 .

The increase in apparent size is not uniform, as already indicated in Fig. 1. Depending on real particle size, the $d_{25}$ image size decreases at one or two positions with distance from the object plane for a short interval, which is due to the peculiarity of diffraction. But, to a good approximation, the overall relation between image diameter and $q_{\text {MIN }}$ can be considered linear throughout the observed parameter range. Therefore, the curves in Fig. 3 are approximated by a linear fit:

$$
d_{25}=A(d) q_{\mathrm{MIN}}+B(d) .
$$

The slope and offset values of these fits are plotted in Fig. 4. These coefficients also exhibit a nearly linear behavior with a nominal particle size that is represented in the following relations:

$$
A(d)=C d+D
$$

and

$$
B(d)=E d+F .
$$

From our measurements the following parameter values are derived:

$$
C=0.962, \quad \mathrm{D}=-10.34 \mu \mathrm{m},
$$

and

$$
E=1.444, \quad \mathrm{~F}=7.036 \mu \mathrm{m} .
$$

Use of these coefficients in (2) provides the linear approximation to the experimental relation between $d_{25}$ and $q_{\text {MIN }}$. These lines are plotted in Fig. 3 to give an indication of the quality of this approximation.

To ensure that the measurements with the spinning glass disk are also valid for real water droplets, a mod- 


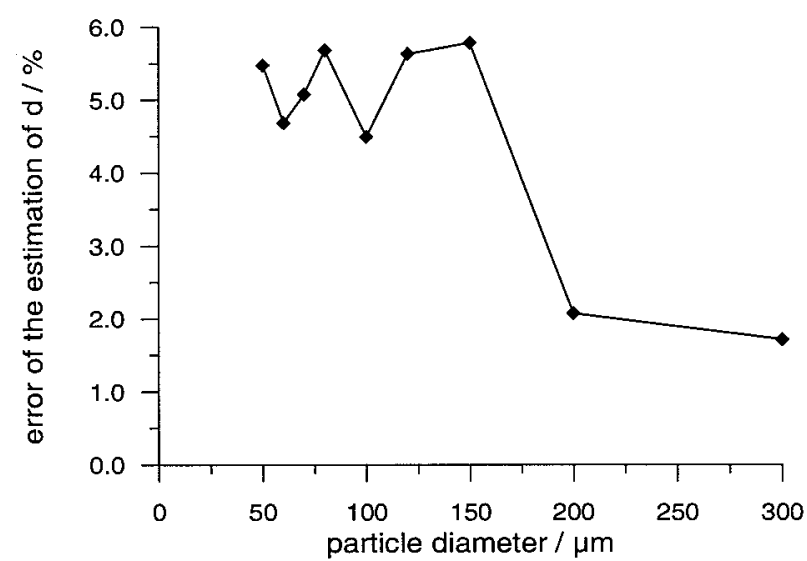

FIG. 5. Error of particle size estimated with (5) vs nominal spot size

ified Hewlett Packard desk jet print cartridge was used to produce water droplets of a diameter of about $60 \mu \mathrm{m}$. Some uncertainty in drop size has to be accepted due to the limits in the repeat quality of this simple device. In addition, the diameter had to be derived from the Grey probe measurements in the object plane, as no drop size specification is available for the print cartridge. The results show the same characteristics as those for the opaque spots (Fig. 3). This supports the assumption that the recorded shadow pattern is mostly due to diffraction processes that are primarily dependent on particle cross section.

Equations (2)-(4) can be solved to obtain the true particle diameter as a function of the apparent diameter $d_{25}$ and the fraction of MIN pixels $q_{\mathrm{MIN}}$ :

$$
d=\frac{d_{25}-D-F q_{\mathrm{MIN}}}{E q_{\mathrm{MIN}}+C} .
$$

To characterize the accuracy of the fit, the diameter was calculated by (5) for 500 valid images for each spot size and positions with $q_{\mathrm{MIN}}<1$. The average value was compared to the nominal spot diameter. Figure 5 shows that the rms deviation of this estimate is less than $6 \%$ from the nominal size.

\section{Depth of field}

Based on the three requirements put forward at the end of section $2 b$, a unique definition of the depth of field became possible. For particle diameters greater than $90 \mu \mathrm{m}$ this depth of field is larger than the distance of $61 \mathrm{~mm}$ between the probe tips, which should only be the case for particles greater than $160 \mu \mathrm{m}$ if (1) would hold. For particles smaller than $90 \mu \mathrm{m}$ a certain distance from the object plane exists at which all pixels are shadowed at the lowest (MIN) grey level (i.e., $q_{\mathrm{MIN}}=1$ ). By increasing the distance from the object plane farther, the image changes significantly, but $q_{\mathrm{MIN}}$ does not, as no pixels at the MID or MAX shadow level are left.

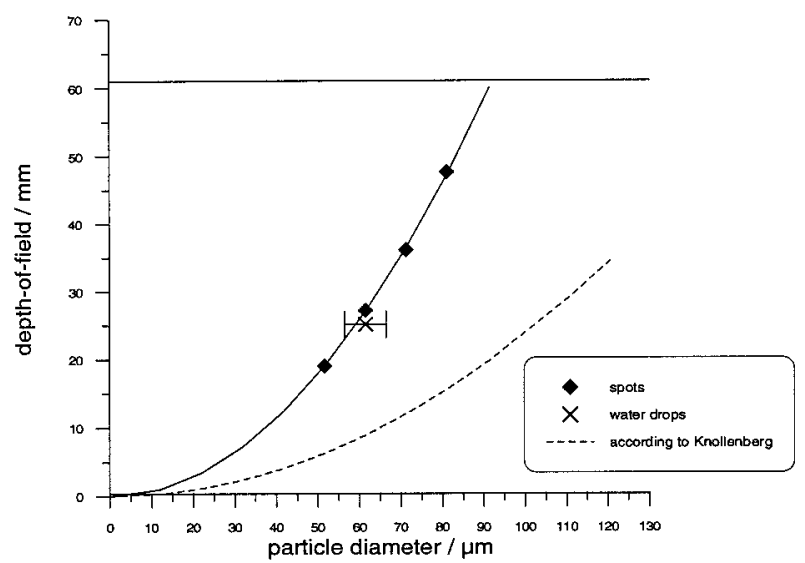

FIG. 6. Depth of field of the 2D-Grey probe vs nominal spot size. The dotted line represents the values from (1) that are normally used in the literature for the evaluation of $2 \mathrm{D}-\mathrm{C}$ data.

Therefore, the minimum distance from the object plane with $q_{\text {MIN }}=1$ would be the appropriate limit for the depth of field in analogy to the hardware criterion of the $2 \mathrm{D}-\mathrm{C}$ probes, which are not able to detect pixels below the $50 \%$ threshold level. Unfortunately, measurement of the exact position of this distance posed some problems, as the number of MIN pixels does not vanish at the same position in all of the spot images. This is probably due to minor differences in spot diameter, the digitization problem when local intensity maxima have a diameter comparable to the image pixel size, and the inhomogeneities in the laser beam. In fact, while most of the spot images lose their MID pixels within a very short distance (cf. Fig. 1), a few others keep MID pixels for quite some additional distance from the object plane. Therefore, we decided to include only cases with at least $2 \%$ of the pixels at the MID shadow level of $q_{\text {MIN }} \leq 0.98$. This corresponds to a minimum number of two MID pixels for $50-\mu \mathrm{m}$ particles and of five for $80-\mu \mathrm{m}$ particles. Due to the rapid drop of the number of MID-level pixels near this limit, the results are not very sensitive to the detailed selection of the limiting $q_{\mathrm{MIN}}$ value.

This $q_{\text {MIN }} \geq 0.98$ threshold defines a depth of field in the sense of the three requirements put forward in section 2 b. Figure 6 contains the measured extent of the so-defined depth of field for different particle sizes. A quadratic dependence on the particle diameter fits the observed data points well for particle sizes from 50 to $90 \mu \mathrm{m}$. For particle sizes greater than $90 \mu \mathrm{m}$ the depth of field of the Grey probe is limited by the mutual distance of the probe tips of $61 \mathrm{~mm}$. Such a quadratic dependence is consistent with theoretical studies of Korolev et al. (1991), as well as with the experimental results of Knollenberg (section 2b). However, the depth of field found by our measurements is three times larger than given by (1). It follows the relation 


$$
l_{\mathrm{DOF}}=\frac{9}{2 \lambda} d^{2} .
$$

According to our definition, the depth of field of the Grey probe is reached when less than $2 \%$ of the pixels are at the MID (50\%) or MAX (75\%) grey level. This is nearly equivalent to the distance from the object plane in the 2D-C probe, where less than one pixel is shadowed at $50 \%$ or higher (cf. section 2b). This depth of field of the $2 \mathrm{D}-\mathrm{C}$ probe was additionally investigated with the help of the described sample glass plate (Reuter 1994). The "total shadow-or" signal of the probe for $50-\mu \mathrm{m}$ spots at different offsets from the object plane was monitored. The measured depth of field was 2.5 times larger than expected by using (1). This coincides again with the Fresnel diffraction modeling results of Korolev et al. (1998).

\section{Discussion}

This work presents a new approach to improved estimation of cloud droplet diameters from 2D-Grey probe data. The apparent diameter $d_{25}$ and the fraction of MIN pixels $q_{\text {MIN }}$ are used to calculate the true size of spherical particles with diameters between 50 and $500 \mu \mathrm{m}$ with the help of an experimentally derived formula. For particle sizes greater than $90 \mu \mathrm{m}$ the depth of field is larger than the distance between the probe tips, which limits the sampling distance along the laser beam. For spherical particles with diameters from 50 to $90 \mu \mathrm{m}$ the depth of field determined using the described retrieval algorithm is shown to be three times larger than the value determined using (1). In addition, it provides further insight into the imaging behavior of OAPs but raises questions concerning the impact of out of focus images on the analysis of $2 \mathrm{D}-\mathrm{C}$ probe data.

Joe and List (1987), who were the first to describe the mis-sizing of out of focus particle images, proposed narrowing the depth of field severely so that significant mis-sizing would no longer occur. But this leads to sampling problems when analyzing small particles, where the images have to be rejected even at small offsets from the object plane. The retrieval algorithm described in the present paper makes, however, full use of the grey-level distribution of each image and enables the analysis of all images with $q_{\text {MIN }}<0.98$ with a remaining rms errors of the derived particle size of less than $6 \%$.

To illustrate the impact of the proposed improvements on the data analysis, an example size spectrum from the European Cloud Radiation Experiment (EUCREX 94) airborne measurement campaign is shown in Fig. 7. This campaign was mainly dedicated to the remote sensing of cloud properties. The measurement flights for Fig. 7 took place on 12 April 1994 to the west of Brittany, France, in a cold frontal multilayer cloud system that approached the coast of Brittany from the northwest. The displayed spectrum was recorded by a standard forward scattering spectrometer probe (FSSP 100) and the
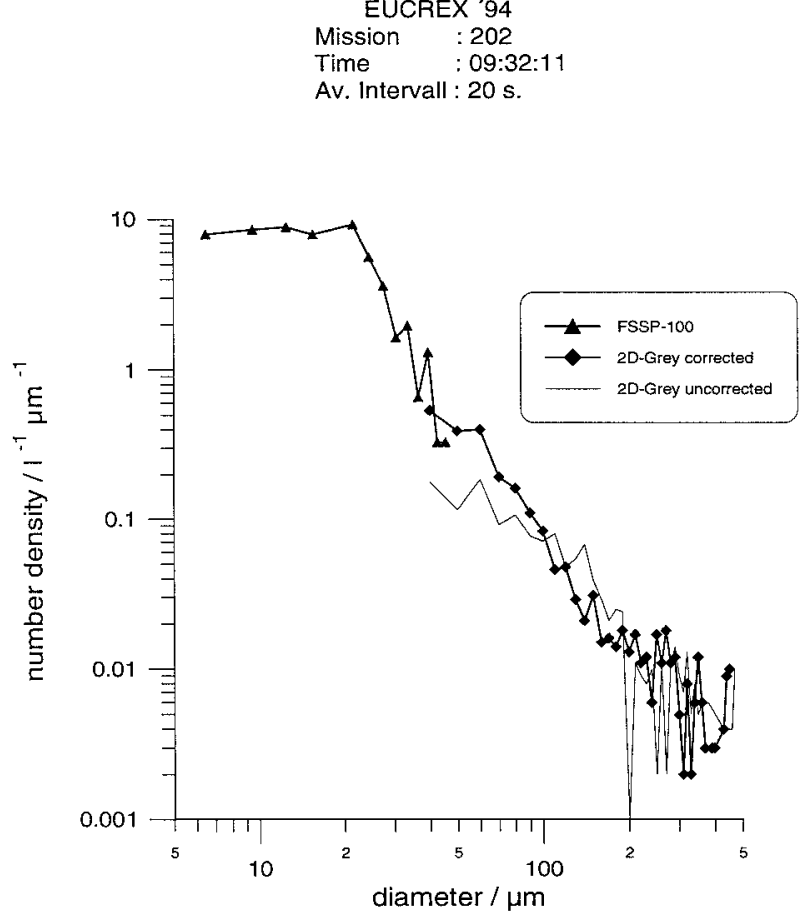

FIG. 7. Example of a droplet size spectrum recorded on 12 April 1994 during EUCREX 94 with an FSSP 100 and a PMS 2D-Grey cloud probe. The Grey probe data are evaluated with the improved procedure of the present paper and, for comparison, without (labeled "uncorrected").

Grey probe used in this study, mounted on the research airplane Merlin IV of Météo-France. The measurements represent $20 \mathrm{~s}$ of a horizontal flight leg at an altitude of about 3000-m height with slightly positive environmental temperature. This example has been chosen for the rather homogeneous conditions and the relatively high concentration of larger droplets. The FSSP size spectrum exhibits a maximum around $20 \mu \mathrm{m}$. An uncorrected size spectrum has been derived from the Grey probe data by assuming that the image diameter for all particles with at least one pixel at the MID shadow level $(50 \%)$ is equal to their real diameter and by calculating the corresponding depth of field from (1). All particles without pixels at the MID shadow level are discarded. This procedure is in analogy with the usual evaluation of 2D-C probe data. For comparison, the procedure proposed in this paper has been applied to the data with an extrapolation to particles smaller than $50 \mu \mathrm{m}$. The 2D spectrum is limited to diameters around $40 \mu \mathrm{m}$; for smaller sizes the reliability of results is limited by statistical problems due to digitization errors. No significant differences are found for droplets larger than 200 $\mu \mathrm{m}$. In the $100-200-\mu \mathrm{m}$ size range oversizing is corrected by the new method and less particles are counted than with the uncorrected method. These corrected particles add to the number of particles smaller than 100 $\mu \mathrm{m}$ and increase their number density. This under- 
counting of smaller and overcounting of larger droplets in the uncorrected spectrum leads to the often-observed change in the gradient of the number density with droplet size at the intersection between FSSP and 2D size spectra (e.g., Martin et al. 1994). The corrected spectrum does not exhibit such a suspicious change but reveals it as an artifact of the missing size correction. The impact of these corrections on bulk quantities such as liquid water content and effective particle radius is less dramatic, but still well expressed.

This calculation suggests that out of focus images have a significant impact on particle data analysis, which should be taken into account for the evaluation of OAP $2 \mathrm{D}$ probe measurements.

It should be clearly stated that there are still certain limitations with the presented retrieval technique.

1) It is based on measurements with spherical spots, whereas the shapes of the existing cloud particles are manifold. Generally, it can be applied only to spherical particles and is therefore only the first step toward a reliable technique for data analysis of OAPs. When coping with nonspherical particles, one has to know the corresponding depth of field, which in this case cannot be simply related to the particle size because there is no unique size value for nonspherical particles. This requires a particle habit detection algorithm to treat particles of different shape individually.

2) The smallest available spot size was $50 \mu \mathrm{m}$. For smaller particles errors resulting from the discrete resolution of the array become more important. Therefore, a new glass disk with spot sizes down to $10 \mu \mathrm{m}$ has been designed for similar future measurements.

3) The presented measurements were done at a speed of about $20 \mathrm{~m} \mathrm{~s}^{-1}$. Response time problems of the electronics occurring at realistic flight speeds were not taken into account.

4) While the 2D-Grey probe for this study has been used at a $10-\mu \mathrm{m}$ resolution most of the $2 \mathrm{D}-\mathrm{C}$ and 2D-Grey cloud probes have a resolution of $25 \mu \mathrm{m}$.
Therefore, the present results can not be applied directly to these probes.

Cooperative efforts have been started to clarify these remaining topics further.

Acknowledgments. The authors want to thank H.-H. Brecht for taking care of the technical aspects of our microphysics probes, F. Albers for sponsoring the additional measurements on a 2D-C probe, J.-L. Brenguier (Météo-France) for incorporating our probes in the Merlin IV during EUCREX 94, and B. Zinecker for patiently editing the manuscript. Also we are indebted to P. Lawson and two anonymous reviewers for their helpful comments and suggestions.

\section{REFERENCES}

Albrecht, B. A., 1989: Aerosols, cloud microphysics, and fractional cloudiness. Science, 245, 1227-1230.

Heymsfield, A. J., and D. Baumgardner, 1985: Summary of a workshop on processing 2D probe data. Bull. Amer. Meteor. Soc., 66, 437-440.

Joe, P., and R. List, 1987: Testing and performance of two-dimensional optical array spectrometer with greyscale. J. Atmos. Oceanic Technol., 4, 139-150.

Knollenberg, R. G., 1970: The optical array: An alternative to scattering or extinction for airborne particle size determination. $J$. Appl. Meteor., 9, 86-103.

Korolev, A. V., S. V. Kuznetsov, Yu. E. Makarov, and V. S. Novikov, 1991: Evaluation of measurements of particle size and sample area from optical array probes. J. Atmos. Oceanic Technol., 8, 514-522.

,,-- J. W. Strapp, and G. A. Isaac, 1998: Evaluation of the accuracy of PMS optical array probes, J. Atmos. Oceanic Technol., 15, 708-720.

Martin, G. M., D. W. Johnson, and A. Spice, 1994: The measurement and parameterization of effective radius of droplets in warm stratocumulus clouds. J. Atmos. Sci., 51, 1823-1842.

Reuter, A., 1994: Derivation of particle size distributions with a 2Dgrey probe (in German). Diploma thesis, Meteorologisches Institut, Universität Hamburg, 149 pp. [Available from Dr. S. Bakan, MPI, Bundesstrasse 55, D-20146 Hamburg, Germany.]

Slingo, A., 1990: Sensitivity of the earth's radiation budget to changes in low clouds. Nature, 343, 49-51.

Stephens, G. L., S.-C. Tsay, P. W. Stackhouse Jr., and P. J. Flatau, 1990: The relevance of the microphysical and radiative properties of cirrus clouds to climate and climatic feedback. J. Atmos. Sci., 47, 1742-1753. 
Copyright of Journal of Atmospheric \& Oceanic Technology is the property of American Meteorological Society and its content may not be copied or emailed to multiple sites or posted to a listserv without the copyright holder's express written permission. However, users may print, download, or email articles for individual use. 